\title{
On the Singularity of Multivariate Hermite Interpolation of Total Degree
}

\author{
Zhongyong Hu, ${ }^{1,2}$ Zhaoliang Meng, ${ }^{1}$ and Zhongxuan Luo ${ }^{1,3}$ \\ ${ }^{1}$ School of Mathematical Sciences, Dalian University of Technology, Dalian 116024, China \\ ${ }^{2}$ School of Mathematics and Statistics, Taishan College, Taian 271021, China \\ ${ }^{3}$ School of Software, Dalian University of Technology, Dalian 116620, China
}

Correspondence should be addressed to Zhaoliang Meng; mzhl@dlut.edu.cn

Received 4 March 2016; Accepted 2 August 2016

Academic Editor: Guang Zhang

Copyright (C) 2016 Zhongyong Hu et al. This is an open access article distributed under the Creative Commons Attribution License, which permits unrestricted use, distribution, and reproduction in any medium, provided the original work is properly cited.

\begin{abstract}
We study the singularity of multivariate Hermite interpolation of type total degree on $m$ nodes with $3+d<m \leq d(d+3) / 2$. We first check the number of the interpolation conditions and the dimension of interpolation space. And then the singularity of the interpolation schemes is decided for most cases. Also some regular interpolation schemes are derived, a few of which are proved due to theoretical argument and most of which are verified by numerical method. There are some schemes to be decided and left open.
\end{abstract}

\section{Introduction}

Let $\Pi^{d}$ be the space of all polynomials in $d$ variables, and let $\Pi_{n}^{d}$ be the subspace of polynomials of total degree at most $n$. Let $\mathscr{X}=\left\{X_{1}, X_{2}, \ldots, X_{m}\right\}$ be a set of pairwise distinct points in $\mathbb{R}^{d}$ and $\mathbf{p}=\left\{p_{1}, p_{2}, \ldots, p_{m}\right\}$ be a set of $m$ nonnegative integers. The Hermite interpolation problem to be considered in this paper is described as follows: Find a (unique) polynomial $f \in \Pi_{n}^{d}$ satisfying

$$
\frac{\partial^{\alpha_{1}+\alpha_{2}+\cdots+\alpha_{d}}}{\partial x_{1}^{\alpha_{1}} \cdots \partial x_{d}^{\alpha_{d}}} f\left(X_{q}\right)=c_{q, \boldsymbol{\alpha}}, \quad 1 \leq q \leq m, 0 \leq|\boldsymbol{\alpha}| \leq p_{q},
$$

for given values $c_{q, \boldsymbol{\alpha}}$, where the numbers $p_{q}$ and $n$ are assumed to satisfy

$$
\left(\begin{array}{c}
n+d \\
d
\end{array}\right)=\sum_{q=1}^{m}\left(\begin{array}{c}
p_{q}+d \\
d
\end{array}\right)
$$

Following $[1,2]$, such kind of problem is called Hermite interpolation of type total degree. The interpolation problem $(\mathbf{p}, \mathscr{X})$ is called regular if the above equation has a unique solution for each choice of values $\left\{c_{q, \boldsymbol{\alpha}}, 1 \leq q \leq m, 0 \leq|\boldsymbol{\alpha}| \leq\right.$ $\left.p_{q}\right\}$. Otherwise, the interpolation problem is singular. As shown in [3], the regularity of Hermite interpolation problem $(\mathbf{p}, \mathscr{X})$ implies that it is regular for almost all $\mathscr{X} \subset \mathbb{R}^{d}$ with $|\mathscr{X}|=m$. Hence, in this paper, we will call $\mathbf{p}$ almost $d$ regular if $(\mathbf{p}, \mathscr{X})$ is regular for some $\mathscr{X} \subset \mathbb{R}^{d}$. Otherwise, we call $\mathbf{p} d$-singular. With no confusion, we also call it almost regular or singular for convenience. If $f$ is a nontrivial polynomial satisfying (1) with zero interpolation condition, we call $f$ a vanishing polynomial with respect to $\mathscr{X}$ and $\mathbf{p}$. Obviously, $(\mathbf{p}, \mathscr{X})$ being singular is equivalent to the existence of a vanishing polynomial of degree no more than $n$.

The research of regularity of multivariate Hermite interpolation is more difficult than Lagrange case, although the latter is also difficult. One of the main reasons is that (2) does not hold in some cases. About the results of multivariate Hermite interpolation, one can refer to [1-10] and the references therein. Most recently, authors [5] made further development and gave complete description for the regularity of the interpolation problem on $m=d+k(k \leq 3)$ nodes, which is an extension of the results mentioned in $[1,2]$. Besides, not any other results appeared for a big number of nodes. This paper is an extension of [5] and we will investigate the singularity of Hermite interpolation for $m=d+k \leq d(d+3) / 2$ with $d \geq 3, k \geq 4$. 
This paper is organized as follows. In Section 2, we consider the singularity of the Hermite interpolation of type total degree and present the main results. In Section 3, we present theoretical proofs for some regular schemes. Finally, in Section 4, we conclude our results.

\section{Singularity of Interpolation Schemes}

In this section, we will investigate the singularity of Hermite interpolation of type total degree and (2) is always assumed to hold. Hermite interpolation of type total degree is affinely invariant in the sense that the interpolation is singular or regular for one choice of nodes. In what follows, we assume $m=d+k \leq d(d+3) / 2$. In this case, since $m<\left(\begin{array}{c}d+2 \\ 2\end{array}\right)$, there must exist a nontrivial quadratic polynomial $Q$ which vanishes at $X_{1}, X_{2}, \ldots, X_{m}$. Also there exists a nontrivial linear polynomial $L$ vanishing at $X_{k+1}, X_{k+2}, \ldots, X_{k+d}$. Given $\widetilde{\mathscr{X}}=\left\{X_{i_{1}}, X_{i_{2}}, \ldots, X_{i_{\tau}}\right\} \subset \mathscr{X}$ and $\widetilde{\mathbf{p}}=\left\{p_{i_{1}}, p_{i_{2}}, \ldots, p_{i_{\tau}}\right\}$, if there are vanishing polynomials with respect to $\widetilde{\mathbf{p}}$ and $\widetilde{\mathscr{X}}$, we will denote by ${ }_{t}^{d}[f] \frac{\widetilde{\mathbf{p}}}{\mathscr{X}}$ one vanishing polynomial of them.

Here we always assume that no interpolation happens at $X_{i_{r}}$ if the $r$ th component $p_{i_{r}}$ of $\widetilde{\mathbf{p}}$ is -1 . Obviously, vanishing polynomials always exist if

$$
\sum_{j=1}^{\tau}\left(\begin{array}{c}
p_{i_{j}}+d \\
d
\end{array}\right)<\left(\begin{array}{c}
t+d \\
t
\end{array}\right),
$$

since the number of the equations is less than the number of the unknowns.

For convenience, we always order $0 \leq p_{1} \leq p_{2} \leq \cdots \leq p_{m}$ with $p_{m} \geq 1$. In [5], authors showed that the inequality

$$
n \geq p_{m}+p_{m-1}+1
$$

must hold if ( $\mathbf{p}, \mathscr{X}$ ) is regular, which gives evaluation of $n$ in (2). The following theorem implies that inequality (4) is very sharp.

Theorem 1. Given $\mathscr{X}=\left\{X_{1}, X_{2}, \ldots, X_{m}\right\}$ and $\mathbf{p}=\left\{p_{1}, p_{2}\right.$, $\left.\ldots, p_{m}\right\}$, if $n>p_{m}+p_{m-1}+1$, then $\mathbf{p}$ is singular.

Proof. We first assume $p_{k}+1 \leq p_{m}$. Then $f=Q^{p_{k}+1} L^{p_{m}-p_{k}}$ is a vanishing polynomial with respect to $\mathscr{X}$ and $\mathbf{p}$, and

$$
\begin{aligned}
\operatorname{deg}(f) & =2\left(p_{k}+1\right)+p_{m}-p_{k} \leq p_{m}+p_{k}+2 \\
& \leq p_{m}+p_{m-1}+2 \leq n .
\end{aligned}
$$

Thus, in this case $\mathbf{p}$ is singular.

$$
\text { If } p_{k}=p_{k+1}=\cdots=p_{m} \text {, then } n>p_{m}+p_{m-1}+1=2 p_{m}+1 \text {. }
$$
Thus $Q^{p_{m}+1}$ is a vanishing polynomial with respect to $\mathscr{X}$ and $\mathbf{p}$, and

$$
\operatorname{deg}\left(Q^{p_{m}+1}\right)=2 p_{m}+2 \leq n .
$$

Collecting two cases, we complete the proof.

$$
\text { Next, we assume } n=p_{m}+p_{m-1}+1 \text {. }
$$

Lemma 2. Given $\mathscr{X}=\left\{X_{1}, X_{2}, \ldots, X_{m}\right\}$ and $\mathbf{p}=\left\{p_{1}, p_{2}, \ldots\right.$, $\left.p_{m}\right\}$, if $p_{k}+1 \leq p_{m-1}$, then $\mathbf{p}$ is singular.
Proof. Let $f=Q^{p_{k}+1} \cdot L^{p_{m}-p_{k}}$. Then $f$ is a vanishing polynomial with respect to $\mathscr{X}$ and $\mathbf{p}$. Moreover

$$
\begin{aligned}
\operatorname{deg}(f) & =2\left(p_{k}+1\right)+p_{m}-p_{k}=p_{m}+p_{k}+2 \\
& \leq p_{m}+p_{m-1}+1=n .
\end{aligned}
$$

This completes the proof.

If $p_{k}+1>p_{m-1}$, we easily get $p_{k}=p_{k+1}=\cdots=p_{m-1}$. The following theorem is due to [5], which will be used in next lemma.

Theorem 3 (see [5]). Assume $m \geq 2$. Given $\mathscr{X}=\left\{X_{1}, X_{2}\right.$, $\left.\ldots, X_{m}\right\}$ and $\mathbf{p}=\left\{p_{1}, p_{2}, \ldots, p_{m}\right\}$, if

$$
p_{1}+p_{2}+\cdots+p_{m}+m \leq n d
$$

then the Hermite interpolation of type total degree is singular.

This theorem implies that there exists a vanishing polynomial of degree no more than $n$ with respect to $\mathbf{p}$ and $\mathscr{X}$ if (8) holds.

Lemma 4. Given $\mathscr{X}=\left\{X_{1}, X_{2}, \ldots, X_{m}\right\}$ and $\mathbf{p}=\left\{p_{1}, p_{2}, \ldots\right.$, $\left.p_{m}\right\}$, if $p_{k}=p_{k+1}=\cdots=p_{m-1}$ and $p_{k-1}+2 \leq p_{m}$, then $\mathbf{p}$ is singular.

Proof. Let $P_{1}=Q^{p_{k-1}+1}$. Then $P_{1}$ together with all of its partial derivatives of order up to $p_{k-1}$ vanishes at the $m$ points. For $d+1$ points $X_{k}, X_{k+1}, \ldots, X_{k+d}$, since

$$
\begin{aligned}
& \left(p_{k}-p_{k-1}-1\right)+\left(p_{k+1}-p_{k-1}-1\right)+\cdots \\
& +\left(p_{m}-p_{k-1}-1\right)+(d+1)=d p_{m-1}+p_{m} \\
& -(d+1) p_{k-1}=d p_{m-1}+p_{m}+(d-1) p_{k-1} \\
& -2 d p_{k-1} \leq d p_{m-1}+p_{m}+(d-1)\left(p_{m}-2\right) \\
& -2 d p_{k-1} \leq d\left(p_{m-1}+p_{m}-2 p_{k-1}-1\right),
\end{aligned}
$$

it follows from Theorem 3 that there exists a polynomial $P_{2}$ with $\operatorname{deg}\left(P_{2}\right) \leq p_{m-1}+p_{m}-2 p_{k-1}-1$, together with all of its partial derivatives of order up to $p_{i}-p_{k-1}-1$ vanishing at $X_{i}$ for $i=k, k+1, \ldots, m$. Let $f=P_{1} P_{2}$. Then, $f$ and all of its partial derivatives of order up to $p_{i}$ vanish at $X_{i}$ for $i=$ $1,2, \ldots, m$, and

$$
\begin{aligned}
\operatorname{deg}(f) & =2\left(p_{k-1}+1\right)+p_{m-1}+p_{m}-2 p_{k-1}-1 \\
& \leq p_{m}+p_{m-1}+1 \leq n
\end{aligned}
$$

Thus, the interpolation is singular.

In what follows, we only need to consider $p_{k}=p_{k+1}=$ $\cdots=p_{m-1}$ and $p_{k-1}+1 \geq p_{m}$, which includes

$$
\begin{gathered}
p_{k-1}+1=p_{k}=\cdots=p_{m}, \quad n=2 p_{m}+1, \\
p_{k-1}=\cdots=p_{m-1}=p_{m}-1, \quad n=2 p_{m}, \\
p_{k-1}=p_{k}=\cdots=p_{m}, \quad n=2 p_{m}+1 .
\end{gathered}
$$


Lemma 5. Given $\mathscr{X}=\left\{X_{1}, X_{2}, \ldots, X_{m}\right\}$ and $\mathbf{p}=\left\{p_{1}, p_{2}, \ldots\right.$, $\left.p_{m}\right\}$, suppose that (11) is satisfied; then Hermite interpolation of type total degree is almost regular if and only if $d=3, k=5$ and

$$
\begin{aligned}
& \left\{p_{1}, p_{2}, \ldots, p_{8}\right\} \\
& \quad=\{p-1, p-1, p-1, p-1, p, p, p, p\} .
\end{aligned}
$$

Proof. Set $p_{k}=\cdots=p_{m}=p(p \geq 1)$; then $p_{k-1}=p-1$ and $n=2 p+1$. We first check (2). Let

$$
\begin{aligned}
& G_{k}(d, p)=\sum_{j=1}^{m}\left(\begin{array}{c}
p_{i}+d \\
d
\end{array}\right)-\left(\begin{array}{c}
n+d \\
d
\end{array}\right) \\
& =\frac{1}{d !}\left(\sum_{j=1}^{k-2} \prod_{i=1}^{d}\left(p_{j}+i\right)+\prod_{i=1}^{d}(p+i-1)\right. \\
& \left.+(d+1) \prod_{i=1}^{d}(p+i)-\prod_{i=1}^{d}(2 p+1+i)\right) .
\end{aligned}
$$

Since $p_{j} \leq p_{k-1}$ for $j=1,2, \ldots, k-2$ and $d+k \leq d(d+3) / 2$, then

$$
\begin{array}{r}
G_{k}(d, p) \cdot d ! \leq(k-1) \prod_{i=1}^{d}(p+i-1) \\
+(d+1) \prod_{i=1}^{d}(p+i)
\end{array}
$$

$$
\begin{aligned}
& -\prod_{i=1}^{d}(2 p+1+i) \\
\leq & \frac{1}{2}(d+2)(d-1) \prod_{i=1}^{d}(p+i-1) \\
& +(d+1) \prod_{i=1}^{d}(p+i) \\
& -\prod_{i=1}^{d}(2 p+1+i):=F(d, p) .
\end{aligned}
$$

We will show that $F(d, p)<0$ implies $F(d+1, p)<0$. Note that

$$
\prod_{i=1}^{d}(p+i-1)=\frac{p}{p+d} \prod_{i=1}^{d}(p+i) .
$$

Thus if $F(d, p)<0$, then

$$
\begin{aligned}
& \prod_{i=1}^{d}(p+i) \\
& \quad<\frac{(p+d) \prod_{i=1}^{d}(2 p+1+i)}{(1 / 2)(d+2)(d-1) p+(d+1)(d+p)} .
\end{aligned}
$$

Hence

$$
\begin{aligned}
F(d+1, p) & =\frac{1}{2}(d+3) d \prod_{i=1}^{d+1}(p+i-1)+(d+2) \prod_{i=1}^{d+1}(p+i)-\prod_{i=1}^{d+1}(2 p+1+i) \\
& =\frac{1}{2}(d+3) d \prod_{i=0}^{d}(p+i)+(d+2) \prod_{i=1}^{d+1}(p+i)-\prod_{i=1}^{d+1}(2 p+1+i) \\
& =\left(\frac{1}{2}(d+3) d p+(d+2)(p+d+1)\right) \prod_{i=1}^{d}(p+i)-\prod_{i=1}^{d+1}(2 p+1+i) \\
& <\frac{((1 / 2)(d+3) d p+(d+2)(p+d+1))(p+d) \prod_{i=1}^{d}(2 p+1+i)}{(1 / 2)(d+2)(d-1) p+(d+1)(d+p)}-\prod_{i=1}^{d+1}(2 p+1+i) \\
& =\frac{\prod_{i=1}^{d}(2 p+1+i)}{(1 / 2)(d+2)(d-1) p+(d+1)(d+p)} \cdot H(d, p),
\end{aligned}
$$

where

$$
\begin{gathered}
H(d, p)=\left(\frac{1}{2}(d+3) d p+(d+2)(p+d+1)\right) \\
\cdot(p+d)-(2 p+d+2) \\
\cdot\left(\frac{1}{2}(d+2)(d-1) p+(d+1)(d+p)\right)
\end{gathered}
$$$$
=-\frac{p}{2}\left(d^{2} p+2 d^{2}+d p-4 p-4\right) .
$$

Since $\partial H / \partial d=-(p / 2)(2 d p+4 d+p)<0$ for $d \geq 2$, $H(d, p)$ is monotonically decreasing about $d$. Thus, $H(d, p)<$ 0 for $d \geq 2$ due to $H(2, p)=-p(p+2)<0$ and $F(d+1, p)<$ 0 . 
Since $F(4, p)=-2 p(p+1)^{2}(p+2)<0$, then $G_{k}(d, p) \leq$ $F(d, p)<0$ for $d \geq 4$, which means that (2) does not hold for $d \geq 4$. Thus we only need to consider $d=3$. In this case

$$
\begin{aligned}
6 G_{k}(3, p) \leq & (k-1) \prod_{i=1}^{3}(p+i-1)+4 \prod_{i=1}^{3}(p+i) \\
& -\prod_{i=1}^{3}(2 p+1+i) \\
= & k p^{3}+3 k p^{2}-5 p^{3}+2 k p-15 p^{2}-10 p \\
= & \begin{cases}-p^{3}-3 p^{2}-2 p<0, & k=4 ; \\
0, & k=5 ; \\
p^{3}+3 p^{2}+2 p, & k=6 .\end{cases}
\end{aligned}
$$

Hence, for $d=3$, (2) does not hold for $k=4$ and holds for $k=$ 5 only if (14) holds. We will show that it is almost regular in next section. For $d=3$ and $k=6$, (2) holds only in the case of $p_{2} \leq p-2$ since (2) holds for $\{p-1, p-1, p-1, p-1, p, p, p, p\}$. We can show that $\mathbf{p}$ is singular if $p_{2} \leq p-2$. In fact, we can take $f=Q^{p-1} \cdot\left({ }_{3}^{3}[f]_{X}^{\widetilde{\mathbf{p}}}\right)$ with $\widetilde{\mathbf{p}}=\{-1,-1,0,0,0,1,1,1,1\}$ as the vanishing polynomial. Obviously $\operatorname{deg}(f)=2(p-1)+3=$ $2 p+1$. The proof is completed.

Lemma 6. Given $\mathscr{X}=\left\{X_{1}, X_{2}, \ldots, X_{m}\right\}$ and $\mathbf{p}=\left\{p_{1}, p_{2}, \ldots\right.$, $\left.p_{m}\right\}$. Suppose that (12) is satisfied and that $p_{k-1}=\cdots=p_{m-1}=$ $p \geq 0$. Then

(i) for $p=0$, (2) holds only for one form $\{\underbrace{0,0, \ldots, 0}_{(1 / 2) d(d+1)}, 1\}$ and Hermite interpolation of type total degree is almost regular for $d \geq 2$;

(ii) for $p=1$, if $d \geq 7$, it is singular; if $3 \leq d \leq 6$, (2) has three positive integer solutions and corresponding interpolation schemes are almost regular;

(iii) for $p \geq 2$,

(a) if $d \geq 5$, it is singular; (b) if $d=4$ and $p>6$, it is also singular;

(c) if $d=4$ and $2 \leq p \leq 6$, (2) has four positive integer solutions and in these cases $\mathbf{p}$ are almost regular;

(d) if $d=3, k=4,6$, it is singular;

(e) if $d=3, k=5$, (2) holds only for one form ( $p-$ $1, p, p, p, p, p, p, p+1)$ and it is almost regular.

Proof. Set $p_{k-1}=\cdots=p_{m-1}=p(p \geq 0)$ and $p_{m}=p+1$; then $n=2 p+2$. If $p=0$, it is easy to check that (2) holds if and only if $m=d(d+1) / 2+1$ and $p_{m}=1, p_{i}=0, i=$ $1,2, \ldots, d(d+1) / 2$. This scheme is almost regular, which will be proved in Theorem 15 of the next section.

For $p \geq 1$, we first check (2). Let

$$
\begin{aligned}
G_{k}(d, p) & =\sum_{j=1}^{m}\left(\begin{array}{c}
p_{i}+d \\
d
\end{array}\right)-\left(\begin{array}{c}
n+d \\
d
\end{array}\right) \\
& =\frac{1}{d !}\left(\sum_{j=1}^{k-2} \prod_{i=1}^{d}\left(p_{j}+i\right)+(d+1) \prod_{i=1}^{d}(p+i)\right. \\
& \left.+\prod_{i=1}^{d}(p+1+i)-\prod_{i=1}^{d}(2 p+2+i)\right) .
\end{aligned}
$$

Since $p_{j} \leq p_{k-1}$ for $j=1,2, \ldots, k-2$ and $d+k \leq d(d+3) / 2$, then

$$
\begin{aligned}
G_{k}(d, p) \cdot d ! \leq & \left(\frac{d(d+3)}{2}-1\right) \prod_{i=1}^{d}(p+i) \\
& +\prod_{i=1}^{d}(p+1+i)-\prod_{i=1}^{d}(2 p+2+i) \\
:= & F(d, p) .
\end{aligned}
$$

By the same argument with Lemma 5, one can show that $F(d, p)<0$ implies that $F(d+1, p)<0$. The following facts can be checked easily:

$$
F(d, p)= \begin{cases}-3(p+4)(p+3)(p+2)(p+1)\left(31 p^{3}+257 p^{2}+440 p-420\right), & d=7 \\ -(p-1)(37 p+150)(p+4)(p+3)(p+2)(p+1), & d=6 \\ -(p+1)(p+2)(p+3)\left(12 p^{2}+23 p-80\right), & d=5 \\ -2(p-6)(p+3)(p+2)(p+1), & d=4\end{cases}
$$

Since $F(7, p)<0$ for $p \geq 1$, (2) does not hold for $d \geq 7$.

For $p=1$ and $3 \leq d \leq 6$, (2) has three positive integer solutions $\{0, \underbrace{1, \ldots, 1}_{6}, 2\}(d=3),\{\underbrace{1, \ldots, 1}_{11}, 2\}(d=4)$ and $\{\underbrace{1, \ldots, 1}, 2\}(d=6)$. These three schemes are almost 26 regular, which can be verified by numerical method; see Remark 7.

Since $F(5, p)<0$ for $p \geq 2$, (2) does not hold for $d \geq 5$ and $p \geq 2$. Similarly, $F(4, p)<0$ for $p>6$ means that (2) does not hold for $d=4$ and $p>6$. 
For $d=4$ and $2 \leq p \leq 6$, (2) has four positive integer solutions

$$
\begin{aligned}
& \{1,1, \underbrace{2, \ldots, 2}_{11}, 3\},\left\{1, \frac{3, \ldots, 3}{12}, 4\right\},\{3, \underbrace{4, \ldots, 4}_{12}, 5\}, \\
& \left\{\frac{6, \ldots, 6}{13}, 7\right\},
\end{aligned}
$$

which are shown to be almost regular by numerical method presented in Remark 7.

Let us consider the case of $d=3$.

From the definition of $G_{k}(d, p)$, we obtain

$$
\begin{aligned}
3 ! \cdot G_{4}(3, p)= & \sum_{j=1}^{2} \prod_{i=1}^{3}\left(p_{j}+i\right)+4 \prod_{i=1}^{3}(p+i) \\
& +\prod_{i=1}^{3}(p+i+1)-\prod_{i=1}^{3}(2 p+2+i) \\
\leq & 6 \prod_{i=1}^{3}(p+i)+\prod_{i=1}^{3}(p+1+i) \\
& -\prod_{i=1}^{3}(2 p+2+i) \\
= & -p(p+1)(p+2)<0 .
\end{aligned}
$$

Then, (2) does not hold for $d=3, k=4$.

For $d=3, k=5$, (2) holds for the form

$$
\{p-1, p, p, p, p, p, p, p+1\} .
$$

Indeed, this form is the only one since $p_{1} \leq p_{2}=p_{3}=p$. This scheme is almost regular, which will be proved in the next section.

Finally, we consider the case of $d=3, k=6$. We can show that $\mathbf{p}$ is singular by taking $Q^{p} \cdot\left({ }_{2}^{3}[f]_{X}^{\mathbf{p}_{1}}\right)$ with $\mathbf{p}_{1}=$ $\{-1,-1,-1,0,0,0,0,0,1\}$ for $p_{3}<p$ and $Q^{p-1} \cdot\left({ }_{4}^{3}[f]_{X}^{\mathbf{p}_{2}}\right)$ with $\mathbf{p}_{2}=\{-1,-1,1,1,1,1,1,1,2\}$ for $p_{3}=p$ as vanishing polynomial with respect to $\mathscr{X}$ and $\mathbf{p}$. Here we use the fact that $p_{2}<p-1$ if $p_{3}=p$ which can be obtained by a simple calculation.

The proof is completed.

Remark 7. Generally speaking, it is difficult to judge the regularity of the interpolation schemes theoretically. For a given $\mathbf{p}$, one possible way to decide the regularity is based on numerical method: calculating the vanishing ideal (see [5] for details) or the corresponding Vandermonde matrix, where the points can be selected randomly. However, the former method needs to do symbolic calculation which is little useful for big $d, m$, and $p$. The latter one needs to judge the singularity of the matrix, which is also difficult if the order is very big. Although so, it is a good way for moderate $d, m$, and $p$, which is employed in this paper for some simple cases.
Lemma 8. Given $\mathscr{X}=\left\{X_{1}, X_{2}, \ldots, X_{m}\right\}$ and $\mathbf{p}=\left\{p_{1}, p_{2}, \ldots\right.$, $\left.p_{m}\right\}$, suppose that (13) is satisfied and that $p_{k-1}=\cdots=p_{m}=$ $p \geq 2$. Then

(i) if $d \geq 21$, (2) never holds;

(ii) if $4 \leq d \leq 20$, (2) has finite positive integer solutions listed in Table 2.

Proof. We first check (2). Let

$$
\begin{aligned}
G_{k} & (d, p)=\sum_{j=1}^{m}\left(\begin{array}{c}
p_{i}+d \\
d
\end{array}\right)-\left(\begin{array}{c}
n+d \\
d
\end{array}\right) \\
= & \frac{1}{d !}\left(\sum_{j=1}^{k-2} \prod_{i=1}^{d}\left(p_{j}+i\right)+(d+2) \prod_{i=1}^{d}(p+i)\right. \\
& \left.-\prod_{i=1}^{d}(2 p+1+i)\right)
\end{aligned}
$$

Since $p_{j} \leq p_{k-1}$ for $j=1,2, \ldots, k-2$ and $d+k \leq d(d+3) / 2$, then

$$
\begin{aligned}
G_{k}(d, p) \cdot d ! \leq & \frac{d(d+3)}{2} \prod_{i=1}^{d}(p+i) \\
& -\prod_{i=1}^{d}(2 p+1+i):=F(d, p) .
\end{aligned}
$$

In the same way with Lemma 5 , one can show that $F(d, p)<0$ implies that $F(d+1, p)<0$ and $F(d, p+1)<0$. By a simple calculation, we have

$$
\begin{gathered}
F(21,2)<0, \\
F(10,3)<0, \\
F(8,4)<0, \\
F(7,5)<0, \\
F(6,6)<0, \\
F(5,8)<0, \\
F(4,20)<0, \\
F(20,2)=0, \\
F(19,2)>0, \\
F(9,3)>0, \\
F(7,4)>0, \\
F(6,5)>0, \\
F(5,6)>0, \\
F(4,8)>0, \\
F(4,19)>0 .
\end{gathered}
$$




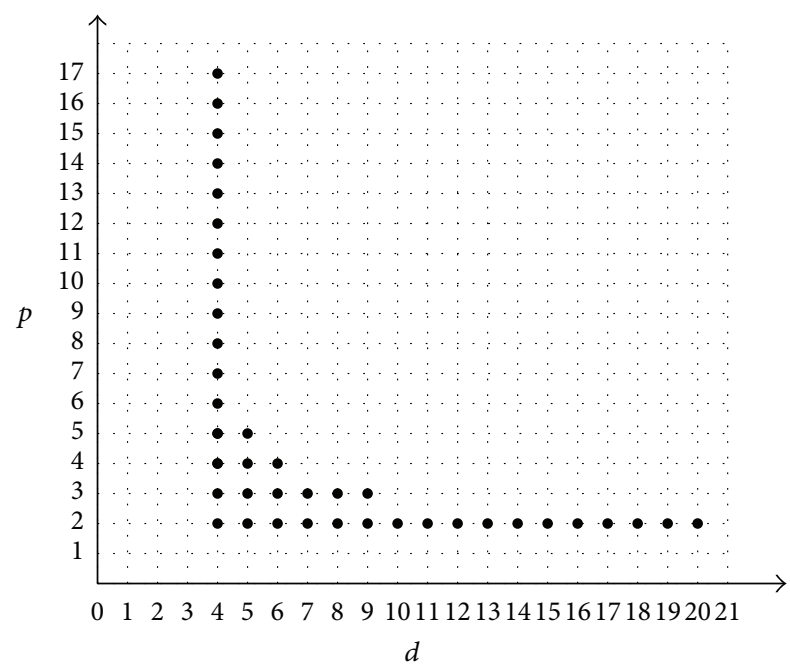

Figure 1: The possible pairs $(d, p)$ satisfying (2) in the case of $d>3$ and $p \geq 2$.

$F(d, p)>0$ implies that $F(\tilde{d}, p)>0$ holds for all $\tilde{d}<d$. Thus we must take $p_{1} \leq p-1$ to ensure (2) if $F(d, p)>0$. Hence, let

$$
\begin{aligned}
\widetilde{F}(d, p)= & \prod_{i=1}^{d}(p-1+i)+\frac{d(d+3)-2}{2} \prod_{i=1}^{d}(p+i) \\
& -\prod_{i=1}^{d}(2 p+1+i)
\end{aligned}
$$

and we again have $\widetilde{F}(d, p)<0$ implying $\widetilde{F}(d+1, p)<0$ and $\widetilde{F}(d, p+1)<0$. It is easy to get

$$
\begin{gathered}
\widetilde{F}(20,2)<0, \\
\widetilde{F}(10,3)<0, \\
\widetilde{F}(7,4)<0, \\
\widetilde{F}(6,5)<0, \\
\widetilde{F}(4,18)<0 .
\end{gathered}
$$

Thus we can obtain the possible pairs $(d, p)$ satisfying (2); see Figure 1.

By detailed analysis and computation, the solution of (2) can be obtained and is listed in Table 2; see Appendix.

Lemma 9. Let $d=3$. Given $\mathscr{X}=\left\{X_{1}, X_{2}, \ldots, X_{m}\right\}$ and $\mathbf{p}=$ $\left\{p_{1}, p_{2}, \ldots, p_{m}\right\}$, suppose that (13) is satisfied and that $p_{k-1}=$ $\cdots=p_{m}=p \geq 2$. Then

(i) for $k=4$, (2) has four positive integer solutions and the corresponding interpolation schemes are singular;

(ii) for $k=5$, (2) has only one positive integer solution and the corresponding interpolation scheme is almost regular; (iii) for $k=6$ and $p<10$, (2) has finite positive integer solutions and all of them are singular.

Proof. (i) $d=3$ and $k=4$.

According to the definition of $G_{k}(d, p)$ in Lemma 8, we obtain

$$
G_{4}(3, p) \leq-\frac{1}{6}(p+1)(p+2)(p-9) .
$$

So, (2) never holds if $p>9$. If $p \leq 9$, (2) has four positive integer solutions $\{2,2,3,3,3,3,3\},\{2,4,4,4,4,4,4\},\{5,6,6,6$, $6,6,6\}$, and $\{9,9,9,9,9,9,9\}$. We claim that they are all singular. To show this we can take

$$
\begin{aligned}
f_{1}= & \left({ }_{4}^{3}[f]_{\mathscr{X}}^{\mathbf{p}_{1}}\right) \cdot\left({ }_{3}^{3}[f]_{\mathscr{X}}^{\mathbf{p}_{2}}\right), \\
f_{2}= & \left({ }_{3}^{3}[f]_{\mathscr{X}}^{\mathbf{p}_{2}}\right) \cdot\left({ }_{3}^{3}[f]_{\mathscr{X}}^{\mathbf{p}_{3}}\right) \cdot\left({ }_{3}^{3}[f]_{\mathscr{X}}^{\mathbf{p}_{4}}\right), \\
f_{3}= & \left({ }_{4}^{3}[f]_{\mathscr{X}}^{\mathbf{p}_{1}}\right) \cdot\left({ }_{4}^{3}[f]_{\mathscr{X}}^{\mathbf{p}_{5}}\right) \cdot\left({ }_{4}^{3}[f]_{\mathscr{X}}^{\mathbf{p}_{7}}\right) \cdot L, \\
f_{4}= & \left({ }_{4}^{3}[f]_{\mathscr{X}}^{\mathbf{p}_{1}}\right) \cdot\left({ }_{4}^{3}[f]_{\mathscr{X}}^{\mathbf{p}_{5}}\right) \cdot\left({ }_{4}^{3}[f]_{\mathscr{X}}^{\mathbf{p}_{6}}\right) \cdot\left({ }_{4}^{3}[f]_{\mathscr{X}}^{\mathbf{p}_{7}}\right) \\
& \cdot\left({ }_{3}^{3}[f]_{\mathscr{X}}^{\mathbf{p}_{2}}\right)
\end{aligned}
$$

as vanishing polynomials with respect to these four solutions, respectively, where

$$
\begin{aligned}
& \mathbf{p}_{1}=\{1,1,2,1,1,1,1\}, \\
& \mathbf{p}_{2}=\{0,0,0,1,1,1,1\}, \\
& \mathbf{p}_{3}=\{0,1,1,1,1,0,0\}, \\
& \mathbf{p}_{4}=\{0,1,1,0,0,1,1\}, \\
& \mathbf{p}_{5}=\{1,2,1,1,1,1,1\}, \\
& \mathbf{p}_{6}=\{1,1,1,2,1,1,1\}, \\
& \mathbf{p}_{7}=\{2,1,1,1,1,1,1\} .
\end{aligned}
$$

(ii) $d=3$ and $k=5$.

Equation (2) has only one positive integer solution $\{0,0,1,2,2,2,2,2\}$ which is almost regular. The regularity can be checked by numerical method mentioned in Remark 7.

(iii) $d=3, k=6$, and $p_{5}=\cdots=p_{9}=p<10$.

We will show that all the schemes in this case are singular. The proof is based on the following three cases.

Case 1. Consider the following: $p_{4}<p$.

(1) $p_{4} \leq p-2$. If (2) holds, then $\mathbf{p}$ is singular. In fact we can check that

$$
Q^{p-2} \cdot\left({ }_{5}^{3}[f]_{\mathscr{X}}^{\mathbf{p}_{8}}\right), \quad \mathbf{p}_{8}=\{0,0,0,0,2,2,2,2,2\}
$$

is the desired vanishing polynomial. 
(2) $p_{4}=p-1$. We consider the case of $p_{3}<p_{4}$ firstly. In this case, (2) holds only if $p_{1} \leq p_{3}-1=p-3$ because

$$
\begin{aligned}
& \left(\begin{array}{c}
p+3 \\
3
\end{array}\right) \cdot 5+\left(\begin{array}{c}
p-1+3 \\
3
\end{array}\right)+\left(\begin{array}{c}
p-2+3 \\
3
\end{array}\right) \cdot 3 \\
& -\left(\begin{array}{c}
2 p+1+3 \\
3
\end{array}\right)=\frac{1}{6}(p+1)\left(p^{2}-4 p+6\right),
\end{aligned}
$$

and we can check that $\mathbf{p}$ is singular if $p_{1} \leq p-4$. In fact, we can take

$$
Q^{p-3} \cdot\left({ }_{7}^{3}[f]_{X}^{\mathbf{p}_{9}}\right), \quad \mathbf{p}_{9}=\{0,1,1,2,3,3,3,3,3\}
$$

as the vanishing polynomial of $\mathbf{p}$.

We next consider the case of $p_{3}=p_{4}$. In this case, $p_{2} \leq$ $p_{3}-2$ must hold; otherwise (2) never holds. If $p_{2}=p_{3}-$ 2 , (2) has two solutions: $\{0,1,3,3,4,4, \ldots, 4\}$ and $\{1,2,4,4$, $5, \ldots, 5\}$. These two schemes are singular, which can be checked by numerical method (see Remark 12). If $p_{2}<p_{3}-2$, then the interpolation scheme $\mathbf{p}$ is singular, which can be shown to take

$$
Q^{p-3} \cdot\left(\begin{array}{l}
3 \\
7
\end{array}[f]_{X}^{\mathbf{p}_{10}}\right), \quad \mathbf{p}_{10}=\{-1,-1,2,2,3,3,3,3,3\}
$$

as the vanishing polynomial. Here the existence of $\left({ }_{7}^{3}[f]_{X}^{\mathbf{p}_{10}}\right)$ follows from the construction of $f_{1}$ in (34).

Case 2. $p_{3}<p_{4}=\cdots=p_{9}=p$.

We first claim that $\mathbf{p}$ is singular if $p_{3} \leq p-3$. Notice that if $p_{3}=p-3$, then (2) holds only if $p_{2}<p_{3}$, which will lead to the singularity of $\mathbf{p}$. The vanishing polynomial can be taken as

$$
\left.Q^{p-3} \cdot\left(\begin{array}{l}
3 \\
3
\end{array} f\right]_{\mathscr{X}}^{\mathbf{p}_{11}}\right) \cdot\left({ }_{2}^{3}[f]_{\mathscr{X}}^{\mathbf{p}_{12}}\right) \cdot\left(\begin{array}{l}
3 \\
2
\end{array}[f]_{\mathscr{X}}^{\mathbf{p}_{13}}\right)
$$

where

$$
\begin{aligned}
& \mathbf{p}_{11}=\{-1,-1,0,0,0,1,1,1,1\}, \\
& \mathbf{p}_{12}=\{-1,-1,-1,1,0,0,0,0,0\}, \\
& \mathbf{p}_{13}=\{-1,-1,-1,0,1,0,0,0,0\} .
\end{aligned}
$$

If $p_{3}=p-2$, then $p_{2}<p_{3}$ must hold to ensure (2). If $p_{3}=p_{2}+1$ or $p_{2}+2$, (2) has four solutions for $p<$ 10: $\{0,3,4,6, \ldots, 6\},\{2,5,6,8, \ldots, 8\},\{1,1,3,5, \ldots, 5\}$, and $\{3,3,5,7, \ldots, 7\}$. These four schemes are singular, which can be checked by numerical method (see Remark 12). If $p_{3} \geq p_{2}+$ 3 , then $\mathbf{p}$ is singular by taking

$$
Q^{p-4} \cdot\left({ }_{3}^{3}[f]_{X}^{\mathbf{p}_{11}}\right) \cdot\left({ }_{3}^{3}[f]_{X}^{\mathbf{p}_{14}}\right) \cdot\left({ }_{3}^{3}[f]_{\mathscr{X}}^{\mathbf{p}_{15}}\right)
$$

as the vanishing polynomial, where

$$
\begin{aligned}
& \mathbf{p}_{14}=\{-1,-1,0,1,1,1,1,0,0\}, \\
& \mathbf{p}_{15}=\{-1,-1,0,1,1,0,0,1,1\} .
\end{aligned}
$$

If $p_{3}=p-1$, then $p_{2}<p_{3}-2$ must hold to ensure (2). In the case of $p_{3}=p_{2}+3$ or $p_{3}=p_{2}+4$, (2) has two solutions for $p<10:\{2,3,7,8, \ldots, 8\}$ and $\{3,4,8,9, \ldots, 9\}$. These two schemes are singular, which can be checked by numerical method (see Remark 12). In the case of $p_{3} \geq p_{2}+5, \mathbf{p}$ is singular, which can be shown by taking

$$
Q^{p-5} \cdot\left({ }_{4}^{3}[f]_{\mathscr{X}}^{\mathbf{p}_{16}}\right) \cdot\left({ }_{4}^{3}[f]_{\mathscr{X}}^{\mathbf{p}_{17}}\right) \cdot\left({ }_{3}^{3}[f]_{\mathscr{X}}^{\mathbf{p}_{18}}\right)
$$

as the vanishing polynomial, where

$$
\begin{aligned}
& \mathbf{p}_{16}=\{-1,-1,1,1,1,1,1,1,2\}, \\
& \mathbf{p}_{17}=\{-1,-1,1,1,1,1,1,2,1\}, \\
& \mathbf{p}_{18}=\{-1,-1,0,1,1,1,1,0,0\} .
\end{aligned}
$$

Case 3. Consider the following: $p_{3}=\cdots=p_{9}$.

To ensure (2), $p_{2}<p-4$ must hold. Furthermore, it is easy to check for $p \leq 10$ and $p_{2}=p-5, p-6$, and $p-7$ that (2) has no solution. If $p_{2} \leq p-8$, then $\mathbf{p}$ is singular and the corresponding vanishing polynomial is taken as

$$
Q^{p^{-7}} \cdot\left({ }_{4}^{3}[f]_{\mathscr{X}}^{\mathbf{p}_{16}}\right) \cdot\left({ }_{4}^{3}[f]_{\mathscr{X}}^{\mathbf{p}_{17}}\right) \cdot\left({ }_{3}^{3}[f]_{\mathscr{X}}^{\mathbf{p}_{18}}\right) \cdot\left({ }_{4}^{3}[f]_{\mathscr{X}}^{\mathbf{p}_{19}}\right),
$$

where

$$
\mathbf{p}_{19}=\{-1,-1,2,1,1,1,1,1,1\}
$$

Thus we complete the proof.

Remark 10. The interpolation scheme $\{9,9,9,9,9,9,9\}$ is first mentioned in [1] and shown to be singular in [11], but one can check that the proof in [11] is not correct. In fact, condition (4.5) in [11] holds with equal sign; hence, its poisedness can not be decided by the necessary condition (4.5) there. The number of the nodes in this case is 7 not 6 .

Remark 11. From the proof in Lemma 9, one can show that the interpolation scheme $d=3$ and $\mathbf{p}=\{3,3,3,3,3,3\}$ is singular by taking

$$
\left({ }_{2}^{3}[f]_{\mathscr{X}}^{\mathbf{p}_{20}}\right) \cdot\left({ }_{2}^{3}[f]_{\mathscr{X}}^{\mathbf{p}_{21}}\right) \cdot\left({ }_{2}^{3}[f]_{\mathscr{X}}^{\mathbf{p}_{22}}\right) \cdot L
$$

where

$$
\begin{aligned}
& \mathbf{p}_{20}=\{1,0,0,0,0,0\}, \\
& \mathbf{p}_{21}=\{0,1,0,0,0,0\}, \\
& \mathbf{p}_{22}=\{0,0,1,0,0,0\} .
\end{aligned}
$$

This scheme was mentioned in $[1,11]$ and wrongly claimed to be almost regular in [5].

Remark 12. The singularity of interpolation schemes $\{0,1$, $3,3,4,4, \ldots, 4\},\{1,2,4,4,5, \ldots, 5\},\{0,3,4,6, \ldots, 6\},\{2,5,6$, $8, \ldots, 8\},\{1,1,3,5, \ldots, 5\},\{3,3,5,7, \ldots, 7\},\{1,6,9,10, \ldots$, $10\},\{2,3,7,8, \ldots, 8\}$, and $\{3,4,8,9, \ldots, 9\}$ can be verified by numerical method. Here we notice that not all of them need 
TABLE 1: The solution of (2) in the case of $p_{k-1}=\cdots=p_{m}=1$.

\begin{tabular}{ccccccccc}
\hline$d$ & $k_{0}$ & $k_{1}$ & $d$ & $k_{0}$ & $k_{1}$ & $d$ & $k_{0}$ & $k_{1}$ \\
\hline 3 & 0 & 5 & 4 & 5 & 6 & 4 & 0 & 7 \\
5 & 8 & 8 & 5 & 2 & 9 & 6 & 14 & 10 \\
6 & 7 & 11 & 6 & 0 & 12 & 7 & 16 & 13 \\
7 & 8 & 14 & 7 & 0 & 15 & 8 & 21 & 16 \\
8 & 12 & 17 & 8 & 3 & 18 & 9 & 30 & 19 \\
9 & 20 & 20 & 9 & 10 & 21 & 9 & 0 & 22 \\
10 & 33 & 23 & 10 & 22 & 24 & 10 & 11 & 25 \\
10 & 0 & 26 & & & & & & \\
\hline
\end{tabular}

to prove independently because we have the following observation:

$\{0,1,3,3,4,4, \ldots, 4\}$ is singular

$\Longrightarrow\{1,2,4,4,5, \ldots, 5\}$ is singular;

$\{0,3,4,6, \ldots, 6\}$ is singular

$\Longrightarrow\{2,5,6,8, \ldots, 8\}$ is singular;

$\{1,1,3,5, \ldots, 5\}$ is singular

$\Longrightarrow\{3,3,5,7, \ldots, 7\}$ is singular;

$\{2,3,7,8, \ldots, 8\}$ is singular

$\Longrightarrow\{3,4,8,9, \ldots, 9\}$ is singular.

Nine nodes can be selected as

$$
\begin{aligned}
& X_{i}=\left(x_{i}, y_{i}, z_{i}\right)^{T}, \quad i=1,2, \ldots, 5, \\
& X_{6}=(1,0,0)^{T}, \\
& X_{7}=(0,1,0)^{T}, \\
& X_{8}=(0,0,1)^{T}, \\
& X_{9}=(0,0,0)^{T} .
\end{aligned}
$$

Lemma 13. Given $\mathscr{X}=\left\{X_{1}, X_{2}, \ldots, X_{m}\right\}$ and $\mathbf{p}=\left\{p_{1}, p_{2}, \ldots\right.$, $\left.p_{m}\right\}$. Suppose that (13) is satisfied and that $p_{k-1}=\cdots=p_{m}=$ $p=1$. Then (2) has finite positive integer solutions for any $d \geq$ 3 and all except one scheme are almost regular.

Proof. By setting $p_{1}=p_{2}=\cdots=p_{k_{0}}=0, p_{k_{0}+1}=\cdots=$ $p_{k_{0}+k_{1}}=1$, and $n=3$ in (2), we obtain

$$
\left(\begin{array}{c}
3+d \\
d
\end{array}\right)=k_{0}\left(\begin{array}{c}
0+d \\
d
\end{array}\right)+k_{1}\left(\begin{array}{c}
1+d \\
d
\end{array}\right)
$$

where $d+2 \leq k_{1} \leq d+k, k_{0}+k_{1}=m$. This equation has finite positive integer solutions for any $d \geq 3$, which is listed in Table 1 for $3 \leq d \leq 10$.

All the schemes except $d=4, k_{0}=0, k_{1}=7$ are almost regular. The singularity of $d=4, k_{0}=0$, and $k_{1}=7$ can be proved by numerical method. The 7 points can be selected as $X_{1}=(0,0,0,0)^{T}, X_{2}=(1,0,0,0)^{T}, X_{3}=(0,1,0,0)^{T}$,
TABLE 2: All the solutions of (2) in Lemma 8.

\begin{tabular}{ccccc}
\hline$d$ & $k$ & $\mathbf{p}$ & $n$ & Singular/regular \\
\hline 4 & \multirow{2}{*}{6} & $(0,1,2,2, \ldots, 2)$ & 5 & Almost regular \\
& & $(2,3,3, \ldots, 3)$ & 7 & Almost regular \\
\hline 4 & 7 & $(1,4,4, \ldots, 4)$ & 9 & \\
\hline & & $(0,1,1,1,1,2,2, \ldots, 2)$ & 5 & Almost regular \\
& & $(1,1,1,3,3, \ldots, 3)$ & 7 & Almost regular \\
& & $(1,2,2,2,3,3, \ldots, 3)$ & 7 & Almost regular \\
4 & 8 & $(2,3,3,4,4, \ldots, 4)$ & 9 & \\
& & $(3,4,5,5,5, \ldots, 5)$ & 11 & \\
& & $(4,6,6, \ldots, 6)$ & 13 & \\
& & $(1,1,1,4,4, \ldots, 4)$ & 9 & \\
& & $(2,3,3,3,3,4,4 \ldots, 4)$ & 9 & \\
& & $(3,3,3,5,5 \ldots, 5)$ & 11 & \\
4 & 9 & $(3,3,6,6 \ldots, 6)$ & 13 & \\
& & $(6,7,8,8, \ldots, 8)$ & 17 & \\
& & $(8,8,9,9,9, \ldots, 9)$ & 19 &
\end{tabular}

$(0,0,0,0,0,0,2,2, \ldots, 2) \quad 5 \quad$ Almost regular $(0,1,1,1,1,1,1,1,2,2 \ldots, 2) \quad 5 \quad$ Almost regular

$(1,1,1,1,2,2,3,3, \ldots, 3) \quad 7 \quad$ Almost regular

$(2,2, \ldots, 2,3,3,3,3,3,3) \quad 7 \quad$ Almost regular

$(1,1,2,2,2,2,2,3,3 \ldots, 3) \quad 7 \quad$ Almost regular

$(1,1,1,3,3,4,4, \ldots, 4) \quad 9$

$(2,3,3,3,3,3,3,4,4, \ldots, 4) \quad 9$

$(1,2,2,2,3,4,4, \ldots, 4) \quad 9$

$410 \quad(1,2,2,4,5,5, \ldots, 5) \quad 11$

$(4,4,4,4,6,6, \ldots, 6) \quad 13$

$(4,5,5,5,5,5,6,6, \ldots, 6) \quad 13$

$(0,3,6,7,7, \ldots, 7) \quad 15$

$(6,7,7,7,7,8,8, \ldots, 8) \quad 17$

$(7,7,7,9,9,9, \ldots, 9) \quad 19$

$(8,9,9,9,10,10,10, \ldots, 10) \quad 21$

$(10,11,11,12,12,12, \ldots, 12) \quad 25$

$(12,13,14,14, \ldots, 14) \quad 29$

$(14,16,16,16, \ldots, 16) \quad 33$

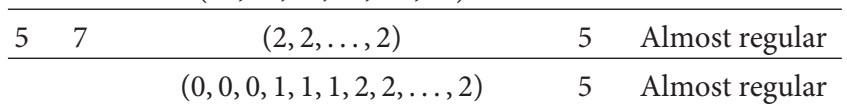

$512 \quad(0,0,1,3,3, \ldots, 3) \quad 7$

$(0,2,2,2,3,3, \ldots, 3) \quad 7$

\begin{tabular}{ccccc} 
& \multicolumn{1}{c}{$(3,3,4,4, \ldots, 4)$} & 9 & \\
\hline 5 & 14 & $(5,6,6, \ldots, 6)$ & 13 & \\
\hline 5 & 15 & $(4,4,6,6, \ldots, 6)$ & 13 & \\
\hline 6 & 12 & $(1,1,2,2, \ldots, 2)$ & 5 & Almost regular \\
\hline 6 & 15 & $(1,1,1,1,1,1,2,2, \ldots, 2)$ & 5 & Almost regular \\
\hline 6 & 17 & $(0,1,2,3,3, \ldots, 3)$ & 7 & \\
\hline 6 & 18 & $(0,0,0,0,0,0,0,1,2,2,2, \ldots, 2)$ & 5 & Almost regular \\
& & $(1,1,1,1,1,1,1,1,1,1,2,2, \ldots, 2)$ & 5 & Almost regular \\
\hline
\end{tabular}


TABLe 2: Continued.

\begin{tabular}{cccc}
\hline$d$ & $k$ & $\mathbf{p}$ & $n$ Singular/regular \\
\hline 6 & 19 & $(0,1,2,2,2,2,3,3, \ldots, 3)$ & 7 \\
\hline 6 & 20 & $(0,1,1,1,1,1,3,3, \ldots, 3)$ & 7 \\
& & $(1,3,3,4,4, \ldots, 4)$ & 9 \\
\hline
\end{tabular}

$(0,0,0,0,0,0,0,1,1,1,1,1,2,2, \ldots, 2,4) 9$

$621 \quad(0,1,2,2,2,2,2,2,2,3,3, \ldots, 3) \quad 7$

\begin{tabular}{lccl} 
& $(\underbrace{1, \ldots, 1}_{14}, \underbrace{2, \ldots, 2}_{13})$ & 5 & Almost regular \\
\hline 7 & 15 & $(2,2, \ldots, 2)$ & 5 \\
\hline 7 & 22 & $(0,0,0,0,1,1,1,1,2,2, \ldots, 2)$ & 5 \\
& & $(1,1,1,1,1,1,1,1,1,2,2, \ldots, 2)$ & 5 \\
\hline 7 & 23 & $(2,2,3,3, \ldots, 3)$ & 7 \\
\hline 8 & 23 & $(1,1,1,2,2, \ldots, 2)$ & 5 \\
\hline 8 & 27 & $(1,1,1,1,1,1,1,1,2,2, \ldots, 2)$ & 5 \\
\hline 8 & 31 & $(0,0,0,0,0,0,0,0,0,1,1,2,2, \ldots, 2)$ & 5 \\
& & $(\underbrace{1, \ldots, 1}_{13}, \underbrace{2, \ldots, 2}_{26})$ & 5 \\
\hline & $(\underbrace{(3, \ldots, 3, \ldots, 3)}_{9}, \underbrace{1, \ldots, 1}_{7}, \underbrace{2, \ldots, 2}_{27})$ & 5 \\
85 & $(\underbrace{1, \ldots, 1}_{18}, \underbrace{2, \ldots, 2}_{25})$ & 5 \\
\hline 9 & 31 & $(0,0,1,1,2,2, \ldots, 2)$ & 5 \\
\hline 9 & 40 & $(\underbrace{0, \ldots, 0}_{7}, \underbrace{1, \ldots, 1}_{7}, \underbrace{2, \ldots, 2}_{35})$ & 5
\end{tabular}

$940 \quad(0,0, \underbrace{1, \ldots, \ldots, 2}_{13,1}) \quad 5$

\begin{tabular}{lll} 
& $\underbrace{}_{13} \underbrace{}_{34}$ & \\
\hline 943 & $(3,3, \ldots, 3)$ & 7 \\
\hline 1048 & $(1,1,1, \underbrace{2, \ldots, 2}_{45})$ & 5 \\
\hline 1048 & $(\underbrace{1, \ldots, 1}_{45}, \underbrace{2, \ldots, 2}_{44})$ & 5 \\
\hline & $(\underbrace{1, \ldots, 1}_{45}, \underbrace{2, \ldots, 2}_{44})$ & 5
\end{tabular}

1048

$$
(\underbrace{1, \ldots, 1}, \underbrace{2, \ldots, 2})
$$

\begin{tabular}{lll}
\hline 1053 & $(\underbrace{0, \ldots, 0}_{11}, \underbrace{1, \ldots, 1}_{8}, \underbrace{2, \ldots, 2}_{44})$ & 5 \\
$(\underbrace{1, \ldots, 1}_{21}, \underbrace{2, \ldots, 2}_{42})$ & 5 \\
\hline 1145 & $(\underbrace{(2, \ldots, 2}_{56})$ & 5 \\
\hline
\end{tabular}

\begin{tabular}{ccc}
\hline $1156 \quad(0,0,0,0,0,0,1,1,1,1,1,1, \underbrace{2, \ldots, 2}_{55})$ & 5 \\
& $(\underbrace{(1, \ldots, 1}_{11}, \underbrace{2, \ldots, 2}_{54})$ & 5 \\
1256 & 5 \\
\hline
\end{tabular}

1379

$$
(0,0,0,0,0,0,0,1,1,1,1, \underbrace{2, \ldots, 2}_{81}) \quad 5
$$

\begin{tabular}{|c|c|c|}
\hline . & $(\underbrace{1, \ldots, 1}, \underbrace{2, \ldots, 2})$ & 5 \\
\hline & $\begin{array}{ll}12 & 80 \\
\end{array}$ & \\
\hline 1492 & $(0,0,0,1,1,1,1,1,1,1, \underbrace{2, \ldots, 2}_{96})$ & 5 \\
\hline 1499 & $(0,0,0, \underbrace{1, \ldots, 1}_{15}, \underbrace{2, \ldots, 2}_{98})$ & 5 \\
\hline 1599 & $(\underbrace{2, \ldots, 2}_{114})$ & 5 \\
\hline \multirow[t]{2}{*}{15114} & $(\underbrace{0, \ldots, 0}_{8}, \underbrace{1, \ldots, 1}_{8}, \underbrace{2, \ldots, 2}_{113})$ & 5 \\
\hline & $(\underbrace{1, \ldots, 1}_{17}, \underbrace{2, \ldots, 2}_{112})$ & 5 \\
\hline
\end{tabular}

TABLE 2: Continued.

\begin{tabular}{lccll}
\hline$d$ & $k$ & $\mathbf{p}$ & $n$ & Singular/regular \\
\hline 16 & 117 & $(2,2, \ldots, 2)$ & 5 & \\
\hline 16 & 125 & $(\underbrace{1, \ldots, 1}_{9}, \underbrace{2, \ldots, 2}_{132})$ & 5 & \\
\hline 16 & 133 & $(\underbrace{1, \ldots, 1}_{18}, \underbrace{2, \ldots, 2}_{131})$ & 5 & \\
\hline 17 & 137 & $\underbrace{2, \ldots, 2}_{154})$ & 5 \\
\hline
\end{tabular}

$X_{4}=(0,0,1,0)^{T}, X_{5}=(0,0,0,1)^{T}, X_{6}=\left(x_{1}^{(1)}, x_{2}^{(1)}\right.$, $\left.x_{3}^{(1)}, x_{4}^{(1)}\right)^{T}$, and $X_{7}=\left(x_{1}^{(2)}, x_{2}^{(2)}, x_{3}^{(2)}, x_{4}^{(2)}\right)^{T}$. Then the Vandermonde matrix is singular by symbolical computation, implying $(1,1,1,1,1,1,1)$ is singular.

Remark 14. In [2], Lorentz presented a conjecture (Conjecture 8) which gives a necessary and sufficient condition about the singularity of multivariate Hermite interpolation. Although the scheme $d=4, k_{0}=0, k_{1}=7, n=3$ is singular, it can not be checked by the conjecture from [2]. Hence Conjecture 8 in [2] is not correct.

\section{The Proof of Regularity of Some Interpolation Schemes}

In this section, we will prove that $\{\underbrace{0,0, \ldots, 0}, 1\}$ is almost $d$ $d(d+1) / 2$

regular and $\{p-1, p, p, p, p, p, p, p+1\}$ and $\{p-1, p-1, p-$ $1, p-1, p, p, p, p\}$ are both almost 3 -regular. To this end, we need to choose $\mathscr{X}$ carefully and then prove the regularity of p.

Theorem 15. Given $\mathscr{X}=\left\{X_{1}, X_{2}, \ldots, X_{m}\right\}$ and $\mathbf{p}=$ $\{\underbrace{0,0, \ldots, 0}, 1\}$, then $\mathbf{p}$ is almost d-regular. $d(d+1) / 2$

Proof. Let $e_{i}=(0,0, \ldots, 0, \stackrel{i}{1}, 0, \ldots, 0)^{T} \in \mathbb{R}^{d}$ and define $X_{i, i}=e_{i}, X_{i, j}=e_{i}+e_{j}$ for $1 \leq i<j \leq d$. Finally, let $X_{m}=(0,0, \ldots, 0)^{T}$ and $\mathscr{X}=\left\{X_{i, j}, 1 \leq i \leq j \leq d, X_{m}\right\}$. We will show the regularity of $\mathbf{p}$. To this end, we assume that $f(X)$ is a polynomial of degree 2 and satisfies all the homogenous interpolation conditions. It only needs to show $f \equiv 0$.

Due to $D^{\alpha} f\left(X_{m}\right)=0$ for $|\alpha| \leq 1, f(X)$ is of form

$$
f(X)=X^{T} A X, \quad A=\left(a_{i j}\right)_{d \times d}, a_{i j}=a_{j i} .
$$

Thus we have

$$
\begin{aligned}
f\left(X_{i, i}\right) & =e_{i}^{T} A e_{i}=a_{i i}=0 \quad(i=1,2, \ldots, d), \\
f\left(X_{i, j}\right) & =\left(e_{i}+e_{j}\right)^{T} A\left(e_{i}+e_{j}\right)=a_{i i}+2 a_{i j}+a_{j j} \\
& =0
\end{aligned}
$$

which lead to $a_{i j}=0$ for $1 \leq i, j \leq d$. Then $f$ should be a zero polynomial, which completes the proof.

To prove the regularity of $\{p-1, p, p, p, p, p, p, p+1\}$ and $\{p-1, p-1, p-1, p-1, p, p, p, p\}$, we need the following lemma. 
Lemma 16 (see $[1,4])$. Let $d=2$; then interpolating the value of a function and all of its partial derivatives of order up to $p$ at each of the three vertices of a triangle as well as the value of the function and all of its derivatives of order up to $p+1 / p-1$ at a fourth point lying anywhere in the interior of the triangle by polynomials from $\Pi_{2 p+2}^{2} / \Pi_{2 p+1}^{2}$, is regular.

In fact the fourth point can lie anywhere except on the three edges of the triangle.

Theorem 17. Let $\mathscr{X}=\left\{X_{1}=(1,1,1)^{T}, X_{2}=(1,0,0)^{T}\right.$, $X_{3}=(0,1,0)^{T}, X_{4}=(0,0,1)^{T}, X_{5}=(1,1,0)^{T}, X_{6}=$ $\left.(1,0,1)^{T}, X_{7}=(0,1,1)^{T}, X_{8}=(0,0,0)^{T}\right\}$, and $\mathbf{p}=\{p-$ $1, p, p, p, p, p, p, p+1\}(p \geq 1)$; then $(\mathbf{p}, \mathscr{X})$ is regular.

Proof. Suppose that $f$ is a polynomial of degree no more than $2 p+2$ such that

$$
\begin{array}{ll}
D^{\alpha} f\left(X_{1}\right)=0, & |\alpha| \leq p-1, \\
D^{\gamma} f\left(X_{8}\right)=0, & |\gamma| \leq p+1, \\
D^{\beta} f\left(X_{i}\right)=0, & |\beta| \leq p, \quad i=2,3, \ldots, 7 .
\end{array}
$$

That is, $f$ satisfies the homogeneous interpolation conditions. To prove this theorem, we only need to show $f \equiv 0$.

Consider the value of $f$ on the plane $x_{3}=0$. It follows from (55) that

$$
\begin{array}{ll}
D^{\gamma} f\left(X_{8}\right)=0, & |\gamma| \leq p+1, \\
D^{\beta} f\left(X_{i}\right)=0, & |\beta| \leq p, \quad i=2,3,5 .
\end{array}
$$

According to Lemma 16, $f$ vanishes on the plane $x_{3}=0$, which implies that $f$ can be divided by $x_{3}$. Similarly, $f$ can be divided by $x_{1}$ and $x_{2}$, respectively. Hence $f$ can be written as $f=x_{1} x_{2} x_{3} f_{1}$ with $\operatorname{deg} f_{1}=2 p-1$.

If $p=1$, by taking $f=x_{1} x_{2} x_{3} f_{1}$ into (55) we obtain

$$
f_{1}\left(X_{i}\right)=0, \quad i=1,5,6,7 .
$$

Thus $f_{1}=0$ and hence $f=0$, which will prove the theorem for $p=1$.

If $p>1$, taking $f=x_{1} x_{2} x_{3} f_{1}$ into (55) will give

$$
\begin{array}{ll}
D^{\alpha} f_{1}\left(X_{1}\right)=0, & |\alpha| \leq p-1, \\
D^{\gamma} f_{1}\left(X_{8}\right)=0, & |\gamma| \leq p-2, \\
D^{\beta} f_{1}\left(X_{i}\right)=0, & |\beta| \leq p-2, i=2,3,4, \\
D^{\mu} f_{1}\left(X_{i}\right)=0, & |\mu| \leq p-1, i=5,6,7 .
\end{array}
$$

Then consider the plane $x_{3}-1=0$. It follows from Lemma 16 and the equations

$$
\begin{aligned}
& D^{\gamma} f_{1}\left(X_{4}\right)=0, \quad|\gamma| \leq p-2, \\
& D^{\beta} f_{1}\left(X_{i}\right)=0, \quad|\beta| \leq p-1, \quad i=1,6,7,
\end{aligned}
$$

that $f_{1}$ can be divided by $x_{3}-1$. Similarly, $f_{1}$ can be also divided by $x_{1}-1$ and $x_{2}-1$, respectively. Thus $f_{1}$ can be written as $f_{1}=\left(x_{1}-1\right)\left(x_{2}-1\right)\left(x_{3}-1\right) f_{2}$ with $\operatorname{deg} f_{2}=2 p-4$.
By collecting the above results, we have $f=x_{1} x_{2} x_{3}\left(x_{1}-\right.$ 1) $\left(x_{2}-1\right)\left(x_{3}-1\right) f_{2}$ with $\operatorname{deg} f_{2}=2 p-4$. If $p=2$, then $f_{2}$ satisfies $f_{2}\left(X_{8}\right)=0$, which implies that $f_{2}=0$ and the theorem will be proved for $p=2$. Otherwise, for $p>2, f_{2}$ satisfies

$$
\begin{array}{ll}
D^{\alpha} f_{2}\left(X_{1}\right)=0, & |\alpha| \leq p-4, \\
D^{\gamma} f_{2}\left(X_{8}\right)=0, & |\gamma| \leq p-2, \\
D^{\beta} f_{2}\left(X_{i}\right)=0, & |\beta| \leq p-3, i=2,3, \ldots, 7,
\end{array}
$$

by taking $f$ into (55).

Clearly, (60) is the same interpolation problem as (55), but with a smaller $p$. Thus we can end the proof by repeating the above process or by induction.

By similar proof, we can get the following theorem and the proof is omitted.

Theorem 18. Let $\mathscr{X}=\left\{X_{1}=(1,1,1)^{T}, X_{2}=(1,0,0)^{T}\right.$, $X_{3}=(0,1,0)^{T}, X_{4}=(0,0,1)^{T}, X_{5}=(1,1,0)^{T}, X_{6}=$ $\left.(1,0,1)^{T}, X_{7}=(0,1,1)^{T}, X_{8}=(0,0,0)^{T}\right\}$, and $\mathbf{p}=\{p-$ $1, p-1, p-1, p-1, p, p, p, p\}(p \geq 1)$; then $(\mathbf{p}, \mathscr{X})$ is regular.

Theorems 17 and 18 imply that $\{p-1, p, p, p, p, p, p, p+1\}$ and $\{p-1, p-1, p-1, p-1, p, p, p, p\}$ are both almost 3 regular.

\section{Conclusion}

In this paper, we consider the singular problem of multivariate Hermite interpolation of total degree. We make a detailed investigation for Hermite interpolation problem of type total degree on $m=d+k \leq(1 / 2) d(d+3)$ nodes in $\mathbb{R}^{d}$.

Given $\mathbf{p}=\left\{p_{1}, p_{2}, \ldots, p_{m}\right\}$ with $m=d+k \leq(1 / 2) d(d+3)$ and $p_{1} \leq p_{2} \leq \cdots \leq p_{m}$, the following results are derived in this paper:

(1) If $p_{k}+1 \leq p_{m-1}$, then $\mathbf{p}$ is singular or (2) does not hold (see Lemma 2).

(2) Suppose $p_{k}=p_{k+1}=\cdots=p_{m-1}$, then

(a) $\mathbf{p}$ is singular if $p_{k-1}+2 \leq p_{m}$; see Lemma 4;

(b) for $p_{k-1}+1=p_{k}=\cdots=p_{m}, n=2 p_{m}+1$, $\mathbf{p}$ is almost regular if and only if $d=3, k=5$, and $\mathbf{p}=\{p-1, p-1, p-1, p-1, p, p, p, p\}$; see Lemma 5; the regularity is given in Theorem 18;

(c) if $p_{k}=p_{k-1}=\cdots=p_{m-1}=p_{m}-1=p-1$, the following 9 interpolation schemes are almost regular (see Lemma 6):

$$
\begin{aligned}
& \{\underbrace{0,0, \ldots, 0}_{(1 / 2) d(d+1)}, 1\}, \\
& \{0, \underbrace{1, \ldots, 1}_{6}, 2\},
\end{aligned}
$$

$$
(d=3),
$$




$$
\begin{gathered}
\{\underbrace{1, \ldots, 1}_{11}, 2\} \quad(d=4), \\
\{\underbrace{1, \ldots, 1}_{26}, 2\} \quad(d=6), \\
\{1,1, \underbrace{2, \ldots, 2}_{11}, 3\} \quad(d=4), \\
\{1, \underbrace{3, \ldots, 3}_{12}, 4\} \quad(d=4), \\
\{3, \underbrace{4, \ldots, 4}_{12}, 5\} \quad(d=4), \\
\{\underbrace{6, \ldots, 6}_{13}, 7\} \quad(d=4), \\
\{p-1, p, p, p, p, p, p, p+1\} \quad(d=3) ;
\end{gathered}
$$

other interpolation schemes are singular;

(d) $p_{k}=p_{k-1}=\cdots=p_{m-1}=p_{m}=p$.

(i) If $p \geq 2$, then (2) never holds for $d \geq 21$ and has finite solutions for $4 \leq d \leq 20$ listed in Table 2 .

(ii) If $p \geq 2, d=3$, and $k=4,5$, only one interpolation scheme $\{0,0,1,2,2,2,2,2\}$ is almost regular and other schemes are singular. If $p \geq 2, d=3$, and $k=6$, (2) has finite solutions for $p<10$ and all of them are singular; see Lemma 9.

(iii) If $p=1$, then (2) has finite solutions listed in Table 1. All except one scheme are almost regular. See Lemma 13 for this case.

Given p, how to decide the regularity theoretically remains difficult and will be our future research project.

\section{Appendix}

\section{Solutions of (2) in Lemma 8}

Table 2 presents all the solutions of (2) in Lemma 8. A small quotient of them is decided by numerical method, but most of them are left open.

\section{Competing Interests}

The authors declare that they have no competing interests.

\section{Acknowledgments}

This project is supported by NNSFC (nos. 11301053, 61432003, 11171052, 11271060, and 61272371) and "the Fundamental Research Funds for the Central Universities."

\section{References}

[1] R. A. Lorentz, Multivariate Birkhoff Interpolation, Springer, 1992.

[2] R. A. Lorentz, "Multivariate Hermite interpolation by algebraic polynomials: a survey," Journal of Computational and Applied Mathematics, vol. 122, no. 1-2, pp. 167-201, 2000.

[3] H. V. Gevorgian, H. A. Hakopian, and A. A. Sahakian, "On the bivariate Hermite interpolation problem," Constructive Approximation, vol. 11, no. 1, pp. 23-35, 1995.

[4] A. Le Méhauté, Interpolation et approximation par des fonctions polynomiales par morceaux dans $\mathrm{R}^{n}$ [Ph.D. thesis], Université de Rennes I, 1984.

[5] Z. Meng and Z. Luo, "On the singularity of multivariate Hermite interpolation," Journal of Computational and Applied Mathematics, vol. 261, pp. 85-94, 2014.

[6] C. de Boor and A. Ron, "On multivariate polynomial interpolation," Constructive Approximation, vol. 6, no. 3, pp. 287-302, 1990.

[7] J. Chai, N. Lei, Y. Li, and P. Xia, “The proper interpolation space for multivariate Birkhoff interpolation," Journal of Computational and Applied Mathematics, vol. 235, no. 10, pp. 3207-3214, 2011.

[8] M. Gasca and T. Sauer, "On bivariate Hermite interpolation with minimal degree polynomials," SIAM Journal on Numerical Analysis, vol. 37, no. 3, pp. 772-798, 2000.

[9] M. Gasca and T. Sauer, "On the history of multivariate polynomial interpolation," Journal of Computational and Applied Mathematics, vol. 122, no. 1-2, pp. 23-35, 2000.

[10] M. Gasca and T. Sauer, "Polynomial interpolation in several variables," Advances in Computational Mathematics, vol. 12, no. 4, pp. 377-410, 2000.

[11] T. Sauer and Y. Xu, "On multivariate Hermite interpolation," Advances in Computational Mathematics, vol. 4, no. 1, pp. 207259, 1995. 


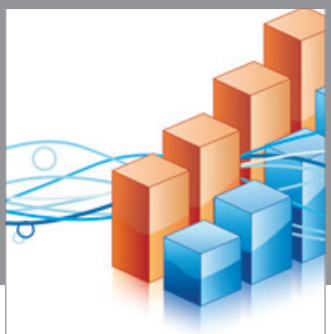

Advances in

Operations Research

vatem alat4

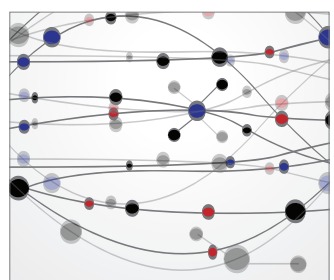

\section{The Scientific} World Journal
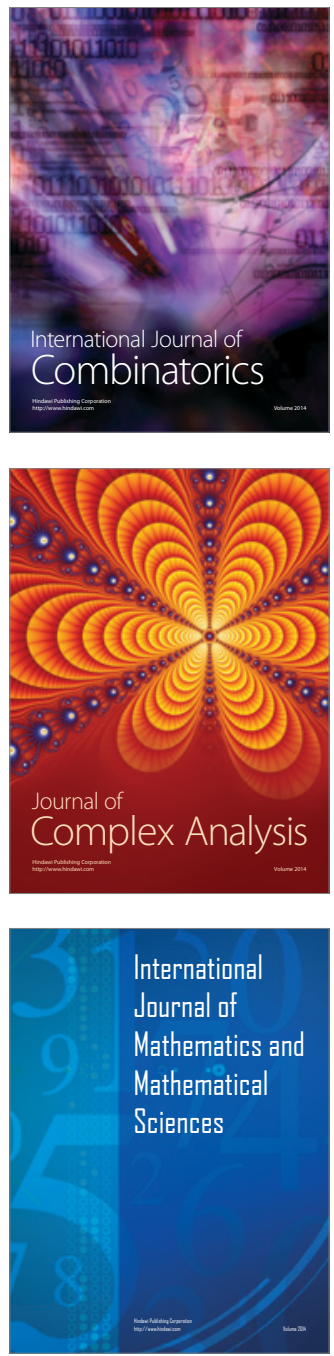
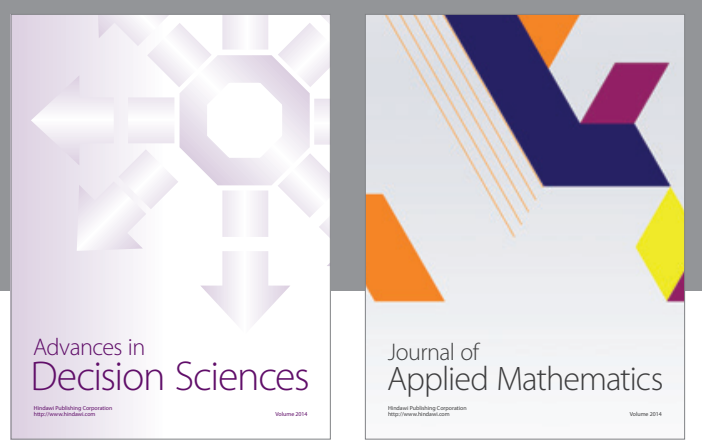

Algebra

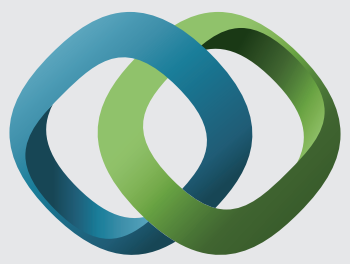

\section{Hindawi}

Submit your manuscripts at

http://www.hindawi.com
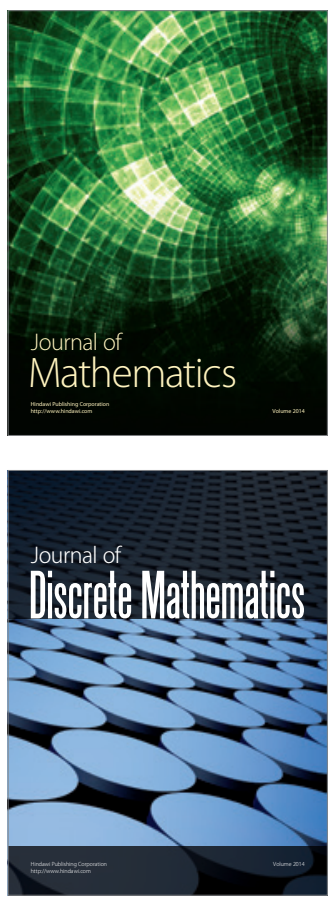

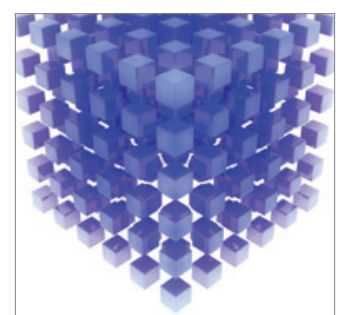

Mathematical Problems in Engineering
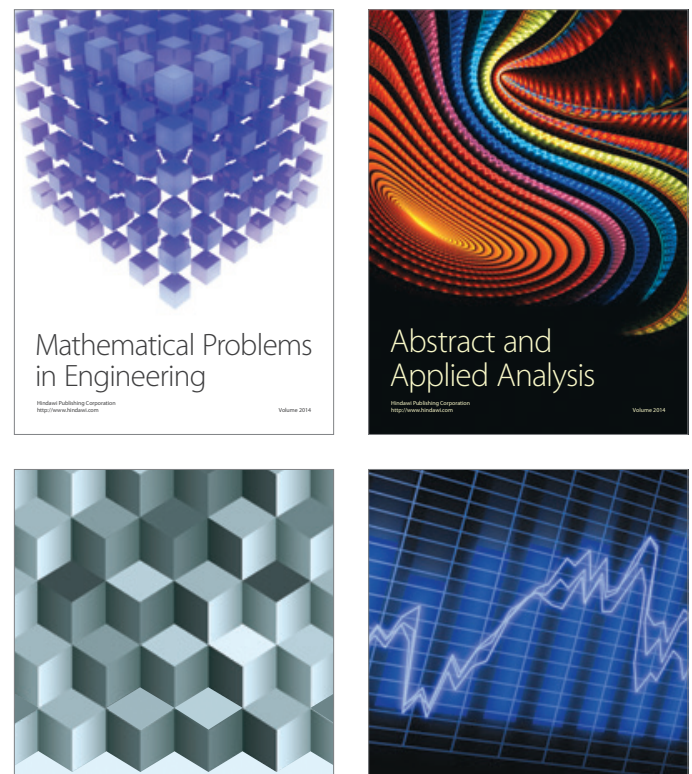

Journal of

Function Spaces

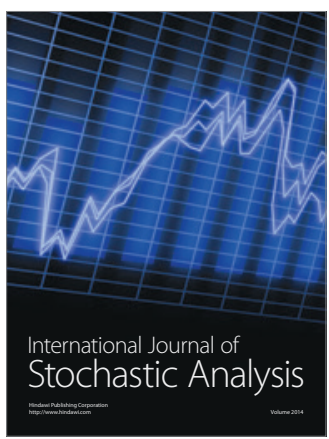

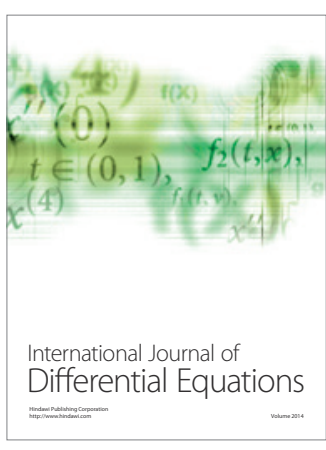
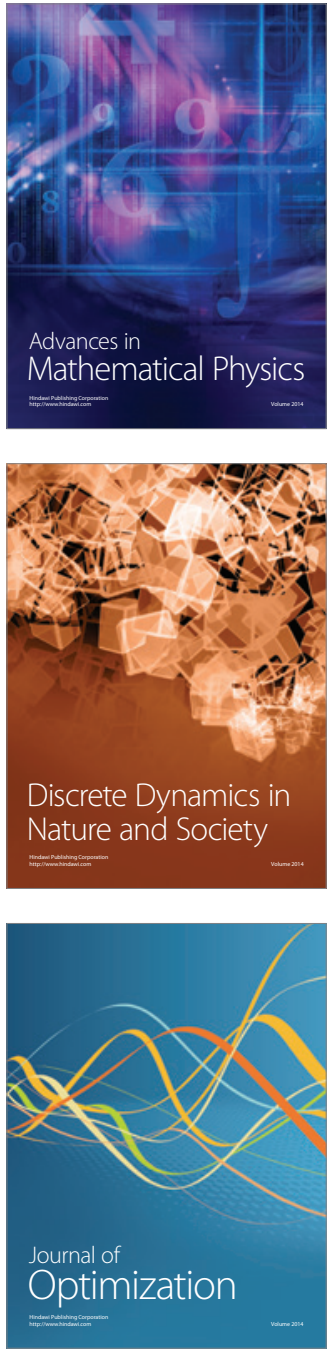